\title{
Transformaciones de la familia conyugal en Chile en el período de la transición democrática (1990-2011)
}

\author{
José Olavarría A.
}

UAHC. Santiago, Chile. Email: jose.olavarria.a@gmail.com

\begin{abstract}
Resumen: ${ }^{1}$ La familia conyugal (matrimonio por voluntad propia, para toda la vida, sustentado en el amor a la pareja, viviendo en un hogar, para procrear y criar juntos los/as hijos: la mujer a cargo de la crianza y las tareas domésticas y el hombre como proveedor y autoridad)estuvo en el centro de las políticas públicas en Chile durante gran parte del siglo XX. Este artículo profundiza en los cambios que se habrían producido en esta configuración familiar en las últimas dos décadas -de transición democrática-, a partir de su contexto histórico, tensiones y conflictos. Para ello se reprocesó, de las bases de datos de las Encuestas CASEN 1990, 2000, 2009 y 2011,la información sobre hogares biparentales con al menos 1 hijo/a, para profundizar sobre esta configuración familiar; tomando los recaudos del caso, porque la CASEN es una encuesta de hogares, no de familias.
\end{abstract}

Palabras clave: Familia, familia conyugal, hogares biparentales, género.

\section{Transformations of the conjugal family in Chile during the democratic transition period (1990-2011)}

\begin{abstract}
The conjugal family (marriage by choice, for life, based on love for the couple, living in a home, to procreate and raise together the children: the woman in charge of raising and of housework, and the male as breadwinner and authority) was at the center of public policy in Chile during much of the twentieth century. This article deepens on the changes occurred in this family configuration in the last two decades of democratic transition, from its historical context, tensions and conflicts. Information was re-processed from data bases of the CASEN Surveys 1990, 2000, 2009 and 2011, the data on two-parent households with at least one child, to deepen the knowledge about this family setting, undertaking the necessary caution, since CASEN is a household survey and not a survey of families.
\end{abstract}

Key words: Family, conjugal family, two-parent households, gender.

\section{Transformações da família conjugal, no Chile, no período de transição democrática (1990-2011)}

Resumo: A família conjugal (casamento por opção, para toda a vida, baseada no amor do casal, morando no lar, para procriar e criar juntos aos filhos/filhas: a mulher no comando da formação e o trabalho doméstico eo homem como provedor e autoridade) esteve no centro das políticas públicas no Chile durante grande parte do século XX. Este artigo explora as mudanças que ocorreram nesta configuração familiar nas últimas duas décadas de transição democrática, a partir de seu contexto 
histórico, tensões e conflitos. Para isso, foram reprocessados os Bancos de dados das Pesquisas CASEN 1990, 2000, 2009 e 2011, com informações sobre as famílias biparentais com pelo menos 1 filho/a, para aprofundar essa configuração familiar, tomando as precauções do caso, porque a Casen é um levantamento dos lares, e não das famílias.

Palavras-chave: Família, Família conjugal, lares biparentais, gênero.

$* * *$

\section{Presentación}

A partir de la revolución industrial, y particularmente en el sector urbano, se produjo la separación de casa y trabajo, del lugar donde se vive y el espacio de la producción; se fue distinguiendo entre lo privado y lo público, que apuntó a separar ámbitos de acción de mujeres y hombres, del poder y del afecto (Jelin 2010). Se fue consolidando así, un tipo particular de familia, la familia conyugal -nuclear y patriarcal- que respondió especialmente a cuatro requerimientos: a) A las políticas de policía de las familias, que buscaron el disciplinamiento de la vida familiar de los sectores pobres urbanos a través de la constitución de familias nucleares con matrimonio civil (Doncelot 1979).b) A las demandas del capitalismo industrial, para asegurar la reproducción de la fuerza de trabajo. c) A las enseñanzas de la Iglesia católica sobre la trilogía familiar, la Sagrada Familia -María, virgen madre; José, carpintero padre, y Jesús, hijo de Dios-, d) al ideal del matrimonio por amor, voluntad e intimidad para toda la vida -que venían desde la Ilustración-, en un hogar propio, para criar juntos a los hijos; ella en lo doméstico y él como proveedor y autoridad.

Los modelos vigentes de familia, paternidad y maternidad -ejes de las identidades y relaciones de género y de los mandatos de ser varón / mujer- surgen, por tanto, en contextos sociales que los hicieron posibles, especialmente a partir del siglo XIX y con fuerza en el siglo XX (Jelin 2010, Doncelot 1979, Stone 1989; Morant y Blufer 1998; Hutchison 1995; Rosenblatt 1995; Romero 1997; Olavarría 2000, 2002; Coontz 2006, Morant y Belufer 1998, Linton 1959², Parsons 1959, Becker 1987). Fueron promovidos activamente, a través de políticas públicas, desde finales del siglo XIX; demandados y apoyados por sectores influyentes en la determinación de la agenda y el uso de recursos públicos, entre ellos, el empresariado del sector fabril y minero emergente, sindicatos y centrales de trabajadores, partidos políticos de la clase media y del movimiento obrero, y la Iglesia Católica.

La familia conyugal fue idealizada como modelo normativo, especialmente en el siglo XX; su reproducción asumida como “normal y natural” y parte constitutiva de la sociedad moderna, a través de la teoría de los roles sexuales (León 1995). En las décadas del 50 y 60, del siglo pasado, esta familia fue centro del debate en Estados Unidos, señalándola como la familia del futuro (Linton 1959; Parsons 1959), la que se requería para una sociedad democrática, urbanizada e industrial, con una considerable igualdad de 
oportunidades (Parsons 1959). Esta familia y este matrimonio, según la visión de Good (1963) “acompañaría la difusión de la industrialización en todo el mundo y reemplazaría la amplia variedad de sistemas matrimoniales y familias propios de otras sociedades tradicionales” (Coontz 2006:317-8).

El fomento de la familia conyugal en Chile, fue la respuesta a la necesidad de asentar e integrar a la creciente población de hombres, trabajadores temporeros, gañanes, que comenzó a 'invadir' las grandes ciudades, especialmente en Santiago en las últimas décadas del siglo XIX. Ellos fueron observados por muchos como una población peligrosa, por las condiciones de vida miserables en las que vivían, generando graves problemas sanitarios y, además, porque sus carencias los podían transformar o los transformaban en delincuentes, para satisfacer sus necesidades de subsistencia. La búsqueda por establecer a estos hombres en un lugar, crear las condiciones para que formaran sus propios núcleos familiares y se hicieran responsables de ellos, se daría en forma paralela a los requerimientos de una emergente demanda de mano de obra, también estable, por parte del capitalismo industrial naciente y los centros mineros que expandían su explotación. Las nuevas factorías requerían de una población trabajadora estable, responsable, que perseverara en el trabajo y tuviese necesidad de conservarlo; que reprodujera en sus propias familias la fuerza de trabajo requerida, en el futuro, por la industria/minería. Estas condiciones se cumplirían por una parte con hombres que se identificaran con su empresa y estuvieran comprometidos y obligados legalmente por el matrimonio civil con una familia, que dependiera de ellos directamente y ante la cual fueran responsables, y por la otra con esposas que se centraran en la reproducción de la familia y lo doméstico (Hutchison 1995; Rosenblatt 1995; Romero 1997). Este modelo permitió asegurar la reproducción de la fuerza de trabajo social y la consolidación de un tipo de familia: la conyugal.

El ordenamiento jurídico existente desde mediados del siglo XIX fue el marco legal en el que se formularon e implementaron las políticas públicas en torno a la familia durante el siglo XX, vigente en sus aspectos principales hasta nuestros días. Las relaciones entre cónyuges y entre padre e hijos estaban (y están) reglamentadas básicamente en el Código Civil, que había incorporado la figura de la familia conyugal en su normativa; cuerpo legal promulgado en 1855 que entró en vigencia en 1857.

A partir de comienzos del siglo XX, cuando las condiciones de vida y de trabajo extremas amenazaron la sobrevivencia de quienes constituían la masa de trabajadores en el país así como de sus familias, el Estado asumió un rol activo en el fortalecimiento de la familia conyugal y su ampliación al conjunto de los núcleos familiares en todo el país. Con el ascenso y expansión de los sectores medios, las políticas macro implementadas desde el Estado apuntaron de distintas maneras a darle sustentabilidad en el tiempo a esta configuración familiar y a sus núcleos que se debatían entre las exigencias sociales de su condición (funcionarios públicos de la administración central, docentes, comerciantes, artesanos, entre otros) y la capacidad económica para la subsistencia y mantención de una calidad de vida acorde. 
Estas políticas reafirmaron una estructura de relaciones al interior de la familia, imponiendo el matrimonio civil por libre voluntad para toda la vida, basado en el amor y con responsabilidades crecientemente específicas tanto a la mujer/madre, en la crianza, acompañamiento de los hijos y las actividades domésticas, como al padre/varón, proveedor, protector y autoridad de su pareja/esposa e hijos/as. Este núcleo familiar, fundamentalmente urbano en sus inicios, permitió la existencia y sustentabilidad de un hogar que tomó distancia del resto de los familiares y lo distinguió de la familia extendida, propia del mundo rural. En estas circunstancias, la estabilidad y permanencia del núcleo familiar conyugal estaba dada por el trabajo remunerado del padre y la dedicación exclusiva o principal de la madre al hogar -para la crianza de los hijos y la mantención de éste-. Si alguno de estos dos actores no podía cumplir su cometido ("rol”) el núcleo entraba en crisis, al no contar con el apoyo directo e inmediato de sus otros familiares, que originalmente estaba presente en la familia extendida.

La consolidación de la familia conyugal y su función en la reproducción social se logró a través de políticas públicas redistributivas de los ingresos, subsidiando bienes y servicios y, especialmente, en el plano de la salud, educación, vivienda, previsión social y en el de las regulaciones y fiscalización del cumplimiento de las normas laborales; políticas públicas que orientaron la acción del Estado hasta la década del 70, en la magnitud que le permitían sus recursos. Se conjugó, así, la ecuación que garantizó la reproducción ampliada de la fuerza de trabajo y la sustentabilidad de esta configuración familiar; la conciliación entre esos espacios descansó en el trabajo no remunerado e invisible de la mujer al interior del hogar y en el salario familiar del varón (Mauro, Godoy y Guzmán 2001; Olavarría 2001).

El golpe de Estado del 73 y las políticas macro, definidas e implementadas por el gobierno cívico-militar, redefinieron el papel del Estado y establecieron un nuevo orden en el ámbito de familia-trabajo que afectó los cimientos que permitían la estabilidad, continuidad y subsistencia de la familia conyugal. Tras el golpe, se inició una profunda transformación del Estado, ahora como Estado subsidiario, expresada en el cambio de las prioridades de las políticas y el uso de los recursos públicos. Los recursos estatales destinados a las políticas sociales se focalizaron en las personas de menores ingresos y se eliminaron las políticas universales. La implementación de la nueva política, con las llamadas modernizaciones, llevó a la modificación no sólo del tamaño del Estado y uso de los recursos públicos, sino también de las reglas de convivencia que habían prevalecido durante el siglo XX. En la década del 2000 se comenzaron nuevamente a aplicar políticas universales, en algunas áreas, y durante el Gobierno de Michele Bachelet se implementaron políticas de Estado para la protección social.

La redefinición de la agenda pública desde 1974, el modo en que se utilizaron los recursos públicos, la política económica de apertura y ajuste estructural, afectaron las bases tanto del orden salarial, como del orden familiar que habían favorecido la existencia de la familia conyugal durante 
gran parte del siglo. La reproducción social de las familias y su sustentabilidad dejaron de ser parte de las políticas del Estado y de la responsabilidad de las empresas privadas/públicas. El ámbito de las familias se privatizó; se perdió el sustento económico que le daban las políticas de conciliación familia-trabajo y quedaron libradas a la capacidad y recursos que disponía cada núcleo familiar. “... la familia ha quedado progresivamente entregada a sí misma, a la autorregulación de sus propios problemas..., se ha ido progresivamente transformando en una organización informal, de convivencia de hecho, a la que la sociedad en su conjunto presta poca atención, y cuya suerte queda entregada a los propios convivientes” (Morandé 1998:79-80).

Pese a la redefinición del Estado, como subsidiario en sus políticas hacia la familia, la familia conyugal persistió, en gran medida, como la familia paradigmática de las políticas públicas durante las últimas décadas del siglo XX y la primera del XXI, aunque ya con algunas diferencias. La significación que tomó para hombres y mujeres la constitución de familia, el trabajo remunerado, y el uso del tiempo, estuvo directamente asociada al tipo de unidad familiar en que se ejerció la conyugalidad /maternidad / paternidad (Fuller 2005, Viveros 2002, Olavarría 2008).

\section{La familia conyugal}

La familia conyugal fue calificada -y lo siguen afirmando muchos/ as- como la máxima expresión de vida familiar en la historia. Fue el logro del ideal presente desde la Ilustración (Morant y Bolufer 1998). "Ha sido la culminación de casi doscientos años de continuos remiendos al modelo de matrimonio basado en el amor y sostenido por el varón protector inventado a finales del siglo XVIII. Ese proceso alcanzó su punto más alto en la década de 1950 -en Estados Unidos y Europa Occidental- con la breve instauración de un estereotipo que desde entonces la gente consideró como el matrimonio tradicional” (Coontz 2006:299). No hay registros en la historia que indiquen que antes "tanta gente haya compartido la experiencia de cortejar libremente a su pareja, casarse por su propia voluntad y establecer su propio hogar. Nunca antes las parejas casadas habían sido tan independientes de los lazos con la familia extendida y con los grupos de su comunidad. Y nunca antes tanta gente había coincidido en definir un único tipo de familia como 'lo normal'”'(Coontz 2006:301).

Pero la familia conyugal, con el matrimonio basado en el amor, se sostiene en bases inestables; señalado así desde el debate de la Ilustración (Morant y Bolufer 1998). Esta configuración familiar quiebra la tradición histórica de familia-matrimonio, donde el matrimonio implicaba establecer/ consolidar una familia política lo más fuerte posible (alianzas), aumentar la fuerza laboral familiar, encontrar una pareja para toda la vida y criar juntos los hijos (Coontz 2006:21).

Ya desde fines de los años veinte del siglo pasado se señalaron aprehensiones acerca del futuro de la familia conyugal, ante las contradic- 
ciones y tensiones, inherentes al matrimonio basado en el amor (Coontz 2006:281). Las mismas reflexiones vuelven a expresarse a fines de la década de los 40:"la opinión pública y el pensamiento erudito estaba dividido sobre lo que depararía la década siguiente. Una escuela de pensamiento sostenía que los valores modernos del individualismo, "principio del placer", expresividad sexual y derechos femeninos desestabilizaría el matrimonio. La otra escuela creía que el matrimonio con el marido encargado del sustento del hogar continuaría siendo la norma cultural y contendría las relaciones sexuales a salvo en seno” (Coontz 2006:294).

Planteado el matrimonio como un contrato civil por amor para toda la viva, se demanda a su vez la posibilidad del divorcio (Coontz 2006). Ya desde la Revolución Francesa se abrió el debate sobre el divorcio, cuando se pone en la agenda legislativa el tema de la reforma del matrimonio y la Constitución (de 1790) seculariza el matrimonio y establece que éste es un contrato civil, un acuerdo entre las partes. La Asamblea Legislativa, el año 1792, aprobó la primera ley de divorcio mutuo, que luego se modificó en sentido restrictivo, se abolió y volvió a restablecerse en los términos originales el año 1975 (Mourant y Bolufer 1998:246).

Quienes teorizaron y fueron los impulsores de la familia conyugal, a mediados del siglo XX, enunciaron sus debilidades y cómo éstas podían afectar su sustentabilidad en el tiempo. Tenían una alta certeza de que el status familiar dependería del estatus ocupacional del marido y del padre, y que la igualdad de oportunidades que tendría el marido y sus hijos era a costa de negar este tipo de igualdad de oportunidades a las mujeres casadas, y de otorgar a los hijos de grupos sociales superiores una considerable ventaja en la competencia por el estatus (Parsons 1959:58).

En los 50 Linton (1959:10) indica que la participación activa de mujeres casadas en el mercado de trabajo productivo hacía ver signos, ya en su tiempo, de "resquebrajamiento de la pauta básica de la interdependencia económica de los sexos; sus consecuencias sobre la institución familiar sólo se perciben, de momento, de manera muy tenue”. En los mismos años Parsons (1959:55) señala que en Estados Unidos la familia conyugal, basada en un acuerdo que tiene como centro los afectos, basada en el amor,“constituye una relación sin ningún apoyo estructural y sin más base, prácticamente, que la atracción emocional: por ello se la ha de proteger contra las tensiones que provoca la competencia por el prestigio entre sus miembros." "Las funciones de esposas y madres son las que predominan, estructuralmente en el tipo familiar. Parece evidente, por ejemplo, que las raíces de la ambivalencia sobre la función femenina son tan profundas que todo intento de obligar o persuadir a la gran mayoría de las mujeres norteamericanas a aceptar el papel de la domesticidad pura y virtuosa, está condenado al fracaso. Si el intento llegase a tener éxito, produciría probablemente, efectos muy perturbadores y crearía más problemas que los que resolvería” (Parsons 1959:64) $)^{3}$. 


\section{El desmoronamiento de la familia conyugal ¿su ocaso?}

Cuando se estimaba posible la consolidación de la familia conyugal y su perdurabilidad en el tiempo comienzan a surgir evidencias de que no sería por un largo tiempo. Paradógicamente, en las últimas décadas, mientras se proclamaba que la familia conyugal era el máximo logro de una configuración familiar acorde con los procesos de modernización y democratización, y de los requerimientos de la economía, ésta comienza a desmoronarse.

Ya desde finales de los 70 en Chile, a partir de las transformaciones que se van constatando en "la familia", investigadores/as de las ciencias sociales se hacen preguntas, investigan y plantean hipótesis sobre "crisis de la familia”. Las evidencias de tensiones y procesos de desinstitucionalización de la familia conyugal son crecientes. Cada vez es más visible que el espacio familiar de esta configuración es afectado por los procesos políticos, culturales y económicos en los que está inserto; asociado en gran medida a los procesos de globalización cultural y económica. La familia, en general, y cada núcleo, en particular, no son inmunes a estos procesos. Procesos que se expanden, a lo menos, en la cultura "occidentalcristiana” y se observan en Chile, en Latinoamérica, en Norteamérica y Europa (Valdés 2008; Valdés y Valdés 2005; Goody 2001; Arriagada 2007; Jelín 2010; García y Oliveira 2006; Fuller 2005; Oyarzún 2005; Durán 2008; Olavarria 2001, 2008, 2009; Coontz 2006, Beck y Beck-Gernsheim 2001, 2012; Wainermann 2005, Estinou 2008, Jelin 2010, Covarrubias et al 1988, Gubbins et al 2003).

Es así que la familia conyugal, logro del siglo XX, ha entrado en un proceso de profundas contradicciones, cambios y resistencias, donde ésta se reformula y reacomoda, se desordena y resignifica; donde el padre y la madre buscan su sitio a partir de la aparición de nuevos valores que postulan la democratización de la vida privada, relaciones horizontales, con proyectos de ambos miembros que buscan su compatibilidad, de mayor intimidad y cercanía afectiva en las relaciones de pareja y con los hijos, y de concordar el uso del tiempo, como consecuencia de la creciente transformación del papel de las mujeres en la familia y la sociedad, y la tendencia a la limitación del poder paterno.

Se hacen visibles las dificultades que existen hoy día para "parentalizarse" en procesos que profundizan las identidades de género y sexuales y que ofrecen resistencias y tendencias a la democratización de la vida privada que desplaza a la familia conyugal moderno-industrial por un modelo relacional.Son más las preguntas que las respuestas sobre las familias, sus actores, el trabajo remunerado, y los hombres y la corresponsabilidad, conciliación y uso del tiempo.

Hombres y mujeres, en proporciones cada vez mayores, aprecian las ventajas de compartir las responsabilidades familiares. Sin embargo, persisten concepciones culturales, estructuras sociales y mentalidades que son 
refractarias a tales cambios y actúan como freno que impide establecer nuevas formas de relaciones que no implique la subordinación de las mujeres (Connell 2009; García y Olivera 2006; Olavarría 2009. Díaz, Godoy y Stecher 2005).Persisten aquellas concepciones culturales sobre la maternidad y la crianza de los hijos en las que la mujer parece ser insustituible e irreemplazable. Ello da cuenta de la tensión que enfrentan las mujeres en la conciliación de su vida familiar, el trabajo al interior del hogar y la posibilidad de acceder a un trabajo en el mercado laboral (SERNAM 2010).

Surgen propuestas, que ponen en entre dicho las bases de la familia conyugal y la división sexual del trabajo, para avanzar en corresponsabilidad -que hombres y mujeres asuman equilibradamente la responsabilidad de satisfacer las necesidades económicas y afectivas de la reproducción social de las familias y los hogares-; para conciliar entre vida laboral, familiar y personal, y lograr corresponsabilidad social en las tareas de cuidado, de manera de vincular la generación de trabajo decente en el corto plazo con efectos en el desarrollo humano y la igualdad de género (OIT 2009:17).

Avanzar en este sentido implica desarticular el entramado de la familia conyugal, confrontarla con las demandas, intereses y derechos que les han sido reconocidos y crecientemente exigen los propios actores -hombre/padre, mujer/madre e hijo/a/s- y con los intereses del capitalismo globalizado y los cambios a la organización del trabajo, en particular los procesos de flexibilización, terciarización y subcontratación. Ello obliga a repensar y debatir sobre la familia en general y la conyugal en particular.

\section{Los datos}

Las encuestas de hogares pueden ser recursos apropiados para el estudio de las familias conyugales. Pero se debe tener en cuenta que no son encuestas de familias. La muestra de la Encuesta CASEN es de hogares ${ }^{4}$; la información que se recoge dice relación con los núcleos familiares ${ }^{5}$ que habitan en una misma vivienda y corresponde a todo tipo de configuraciones familiares de personas que habiten esa vivienda, entre ellas las nucleares biparentales con hijo/a/s.

La caracterización que hace Parsons de la familia conyugal, en algún sentido, puede servir para los fines de establecer una definición operacional de familia conyugal que permita reprocesar la información de las bases de datos de la CASEN. "La familia conyugal típica vive en un hogar separado de los padres de los dos cónyuges. La familia conyugal aislada es importante, porque constituye la unidad doméstica normal. Esto significa que es la unidad de residencia, la unidad cuyos miembros aseguran en común la base económica -especialmente los ingresos monetarios.”(Parsons 1959:39).

De los atributos de la familia conyugal (matrimonio por voluntad propia, para toda la vida, sustentado en el amor a la pareja, viviendo en un hogar, para procrear y criar juntos los/as hijos: la mujer a cargo de la crianza 
y las tareas domésticas y el hombre como proveedor) es posible rescatar los siguientes atributos (variables) de la CASEN: jefatura de hogar, convivencia de pareja con hijo/a/s, matrimonio, y vivienda común. Es indudable, que la definición operacional que se construya a partir de estas variables tiene importantes limitaciones para estudiar la familia conyugal, pero es la que permitiría una mejor aproximación a conocer sobre este tipo de familia a un nivel macrosocial y, de alguna manera, su comparación con otros tipos de configuraciones familiares.

A partir de las variables indicadas (la serie de la Encuesta CASEN repite en los años las preguntas atingentes y sus respuestas), se desagregó en dos subconjuntos la información de la Encuesta, en sus mediciones de los años 1990, 2000, 2009 y 2011, tomando como base la jefatura de hogar: de los hogares biparentales con al menos un hijo/a/s, y del conjunto de los hogares.

El análisis de la familia conyugal se hace desde la definición operacional definida: hogar biparental en el que la pareja conviven con al menos un hijo/a en la misma vivienda. Cada hogar tiene un/a jefe de hogar. Se distinguió los hogares a partir de la información del jefe de hogar y la relación que tenían los miembros de éste con el/a jefe. Para este artículo, el año base es 1990 (CASEN 1990), cuando se recupera la democracia y comienza el período que se ha denominado transición democrática, se compara esa información con las de los años 2000, 2009 y 2011 (CASEN 2000, CASEN 2009 Y CASEN 20116).

Se compara la información obtenida del conjunto de hogares con la de los hogares biparentales con al menos un hijo/a, a partir, por tanto, de la información de los jefes de hogar. Se analiza la información de cada conjunto.

\section{Cantidad de hogares en su conjunto y biparentales con al menos un hijo/a}

En el período bajo estudio -1990-2011- el número de hogares creció en más del 50\%, a partir del año base (1990). El conjunto de hogares aumentó de 3.180.536 a 4.966.890, en valores relativos el incremento fue del 56,2\% (Cuadro $\mathrm{N}^{\circ} 1$ ).

Se observan variaciones, asimismo, en el número de hogares biparentales, con al menos un hijo. El incremento fue de 2.039.832, el año 1990, a 2.361.632, el 2011, con una expansión relativa del 15,8\%. Pese al incremento de estos hogares, es llamativamente menor al del conjunto de los hogares (Cuadro $\mathrm{N}^{\circ} 1$ ).

En el año 1990 los hogares biparentales representaron a casi dos tercios de la totalidad de los hogares (64,1\%). En el año 2000 ese porcentaje alcanzó el 61,6\% del total de hogares. En los años 2009 y 2011 siguió la tendencia a la disminución en los biparentales con al menos 1 hijo/a, a $52,3 \%$ y 47,5\% respectivamente. En dos décadas, el peso específico de 
estos hogares pasó desde casi dos tercios del conjunto de todos los hogares a menos de la mitad (Cuadro $\mathrm{N}^{\circ} 1$ ).

\section{Cuadro $N^{\circ} 1$ \\ Hogares, biparentales y todos los hogares, por sexo del jefe de hogar según año de medición 1990-2011 (CASEN)}

\begin{tabular}{|l|c|c|c|c|c|}
\hline & \multicolumn{2}{|l|}{ Hogares biparentales } & \multicolumn{2}{l|}{ Todos los hogares } & $\begin{array}{c}\text { Relación hogares biparentales } \\
\text { con todos los hogares }\end{array}$ \\
\hline & Total & $\%$ & Total & $\%$ & $\%$ \\
\hline CASEN 1990 & 2.039 .832 & 100,0 & 3.180 .536 & 100,0 & 64,1 \\
\hline CASEN 2000 & 2.404 .892 & 117,9 & 3.901 .612 & 122,7 & 61,6 \\
\hline CASEN 2009 & 2.448 .385 & 120,0 & 4.685 .490 & 147,3 & 52,3 \\
\hline CASEN 2011 & 2.361 .632 & 115,8 & 4.966 .890 & 156,2 & 47,5 \\
\hline
\end{tabular}

La jefatura de hogar por sexo también muestra variaciones en estas dos décadas. En este período (1990-2011) disminuyó la proporción de hombres jefes de hogares del conjunto de los hogares y de los hogares biparentales, y se incrementó la participación de las mujeres.

Para el conjunto de los hogares, los hombres representaron el año 1990 el 79,8\% de las jefaturas, valor que fue disminuyendo en este período hasta el 61,2\% el año 2001. Las mujeres jefas de hogar se incrementaron en la proporción que disminuyen los hombres, pasando de un quinto de las jefaturas a más de un tercio. Es así que el año 1990 equivalían al 20,2\% y el 2011 el 38,8\% (Cuadro N²).

\section{Cuadro $N^{\circ} 2$ \\ Jefatura del conjunto de los hogares por sexo del jefe de hogar según año de medición 1990.2011 CASEM}

\begin{tabular}{|l|r|r|r|l|l|l|}
\hline & \multicolumn{1}{|l|}{ Hombre } & \% & \multicolumn{1}{|c|}{ Mujer } & \% & Total & \% \\
\hline CASEN 1990 & 2.538 .948 & 79,8 & 641.588 & 20,2 & 3.180 .536 & 100,0 \\
\hline CASEN 2000 & 2.996 .752 & 76,8 & 904.860 & 23,2 & 3.901 .612 & 122,7 \\
\hline CASEN 2009 & 3.134 .865 & 66,9 & 1.550 .625 & 33,1 & 4.685 .490 & 147,3 \\
\hline CASEN 2011 & 3.041 .346 & 61,2 & 1.925 .544 & 38,8 & 4.966 .890 & 156,2 \\
\hline
\end{tabular}

Para los hogares biparentales con al menos un hijo, se constata, asimismo, variaciones en las jefaturas según el sexo de quien es reconocido como jefe/a. En el año 1990 casi la totalidad de los jefaturas de hogar correspondió a un hombre $(97,8 \%)$, valor que disminuyó hasta llegar al $84,3 \%$, el 2011. Las mujeres, por su parte, la incrementaron en estas dos décadas, desde una presencia muy baja el año 1990 (2,2\%) al 15,7\% el 2011; el incremento fue de aproximadamente 6 veces para la mujeres jefas de hogar. (Cuadro $\mathrm{N}^{\circ} 3$ ). 


\section{Cuadro $N^{\circ} 3$ \\ Jefatura de hogares biparentales con al menos 1 hijo/a por sexo según año de medición 1990-2011 CASEN}

\begin{tabular}{|l|l|l|l|l|l|l|}
\hline & Hombre & $\%$ & Mujer & $\%$ & Total & $\%$ \\
\hline CASEN 1990 & 1.994 .800 & 97,8 & 45.032 & 2,2 & 2.039 .832 & 100,0 \\
\hline CASEN 2000 & 2.292 .804 & 95,3 & 112.088 & 4,7 & 2.404 .892 & 117,9 \\
\hline CASEN 2009 & 2.161 .073 & 88,3 & 287.312 & 11,7 & 2.448 .385 & 120,0 \\
\hline CASEN 2011 & 1.990 .643 & 84,3 & 370.989 & 15,7 & 2.361 .632 & 115,8 \\
\hline
\end{tabular}

Al comparar la jefatura de hogar por sexo, de los hogares biparentales, con el conjunto de los hogares, se observa la misma tendencia señalada más arriba. Los hombres jefes de hogar biparental con al menos un hijo pasaron de ser algo más de tres cuartas partes a menos de dos tercios entre el año 1990 (78,6\%) y el 2011 (65,5\%) (Cuadro N 4).

En cambio, la proporción de mujeres jefas de hogar de hogares biparentales con al menos un hijo/a casi triplicó su crecimiento en estas dos décadas, en relación al conjunto de mujeres jefas de hogar, pasando del 7,0\% el año 1990 al 19,3\% el 2011 (Cuadro N 4).

\section{Cuadro $N^{\circ} 4$ \\ Jefatura de hogar biparentales con al menos 1 hijo/a en relación al conjunto de hogares por sexo según año de medición 1990-2011 CASEN}

\begin{tabular}{|c|c|c|}
\hline & $\begin{array}{r}\text { \% Jefes de hogar biparentales en relación } \\
\text { a todos los hogares por sexo }\end{array}$ \\
\hline & \% Hombres & \% Mujeres \\
\hline CASEN 1990 & 78,6 & 7,0 \\
\hline CASEN 2000 & 76,5 & 12,4 \\
\hline CASEN 2009 & 68,9 & 18,5 \\
\hline CASEN 2011 & 65,5 & 19,3 \\
\hline
\end{tabular}

\section{Estado civil de los jefes de hogar Casados/as}

En las dos décadas pasadas se observan cambio importantes en el estado civil de los/as jefes de hogares, tanto del conjunto de hogares como de hogares biparentales con al menos un hijo/a. La proporción de casados/ as decreció sistemáticamente entre 1990 y 2011. En cambio, se incrementó la proporción en todas las otras categorías; a saber, convivientes, anulados, separados, divorciados, viudos y solteros.

Para el conjunto de hogares en Chile, el año 1990, los jefes de hogar casados/as representaban dos tercios (68,8\%), valor que descendió hasta el 46,5\% el año 2011. Los/as convivientes pasaron del 6,3\% al 15\%; los/as 
anulados/as, separados/as, divorciados/as o viudos subieron del $18,2 \%$ al 23,3\%, y los/as solteros/as del 6,7\% al 15,2\% (Cuadro $\mathrm{N}^{\circ}$ 5).

\section{Cuadro $N^{\circ} 5$ \\ Jefe de hogar por estado civil según año de medición. \\ Todos los hogares \\ 1990-2011 (CASEN)}

\begin{tabular}{|l|c|c|c|c|c|c|c|c|c|c|}
\hline & Casados & $\%$ & Convivientes & \% & $\begin{array}{c}\text { Anulado/a, } \\
\text { separado/a, } \\
\text { divorciado/a, } \\
\text { viudo/a }\end{array}$ & \% & Soltero/a & \% & Total & $\%$ \\
\hline CASEN 1990 & 2.189 .071 & 68,8 & 201.544 & 6,3 & 578.182 & 18,2 & 211.739 & 6,7 & 3.180 .536 & 100,0 \\
\hline CASEN 2000 & 2.409 .133 & 61,7 & 426.763 & 10,9 & 728.612 & 18,7 & 337.104 & 8,6 & 3.901 .612 & 100,0 \\
\hline CASEN 2009 & 2.431 .644 & 51,9 & 666.536 & 14,2 & 1.025 .495 & 21,9 & 561.815 & 12,0 & 4.685 .490 & 100,0 \\
\hline CASEN 2011 & 2.310 .198 & 46,5 & 745.210 & 15,0 & 1.155 .159 & 23,3 & 756.323 & 15,2 & 4.966 .890 & 100,0 \\
\hline
\end{tabular}

Al iniciar el período -1990- los/as jefes de hogar casados en los hogares biparentales con al menos un hijo constituían el 92\%; a lo largo de las dos décadas su proporción disminuyó al 76,4\% el año 2011 (Cuadro $\mathrm{N}^{\circ} 6$ ).

\section{Cuadro $\mathrm{N}^{\circ} 6$}

Jefe de hogar por estado civil según año de medición. Hogares biparentales con al menos 1 hijo/a 1990-2011 (CASEN)

\begin{tabular}{|l|c|c|c|c|c|c|}
\hline & Casados & $\%$ & Convivientes & \% & Total & \% \\
\hline CASEN 1990 & 1.876 .293 & 92,0 & 163.539 & 8,0 & 2.039 .832 & 100,0 \\
\hline CASEN 2000 & 2.062 .279 & 85,8 & 342.613 & 14,2 & 2.404 .892 & 100,0 \\
\hline CASEN 2009 & 1.931 .636 & 78,9 & 516.749 & 21,1 & 2.448 .385 & 100,0 \\
\hline CASEN 2011 & 1.803 .978 & 76,4 & 557.654 & 23,6 & 2.361 .632 & 100,0 \\
\hline
\end{tabular}

Pero la tendencia a la disminución de casados, para el conjunto de los y las jefes de hogar, tiene expresiones contrapuestas entre hombres y mujeres durante estas dos décadas: crece la proporción de mujeres jefas de hogar casadas tanto para el total de los hogares, como para los hogares biparentales con al menos un hijo/a, y decrece la de hombres. Para el total de los hogares los hombres jefes de hogar casados disminuyen del 84,1\%, el año 1990, al 65,7\% el 2011. Las mujeres jefas de hogar casadas, en cambio, aumentan del 8,4\% al 16,2\%; prácticamente su duplican (Cuadro $\mathrm{N}^{\circ} 7$ ). 


\section{Cuadro N ${ }^{\circ} 7$ \\ Jefes de hogar casados/as por sexo según año de medición. \\ Todos los hogares \\ 1990-2011 (CASEN)}

\begin{tabular}{|c|c|c|c|c|c|c|}
\hline \multirow{2}{*}{ CASADOS/AS } & \multicolumn{4}{|c|}{ Sexo } & \multirow{2}{*}{ Total } & \multirow{2}{*}{$\%$} \\
\cline { 2 - 5 } & Hombre & $\%$ & Mujer & $\%$ & & \\
\hline CASEN 1990 & 2.134 .883 & 84,1 & 54.188 & 8,4 & 2.189 .071 & 68,8 \\
\hline CASEN 2000 & 2.324 .394 & 77,6 & 84.739 & 9,4 & 2.409 .133 & 61,7 \\
\hline CASEN 2009 & 2.189 .669 & 69,8 & 241.975 & 15,6 & 2.431 .644 & 51,9 \\
\hline CASEN 2011 & 1.998 .208 & 65,7 & 311.990 & 16,2 & 2.310 .198 & 46,5 \\
\hline
\end{tabular}

La misma tendencia de constata entre los/as jefes de hogares biparentales con al menos un hijo/a; se incrementa seis veces la proporción de mujeres casadas, entre los/as casados, y decrece el 11,2\% la de los varones (Cuadro $\mathrm{N}^{\circ} 8$ ).

\section{Cuadro $\mathrm{N}^{\circ} 8$ \\ Jefes de hogar casados/as por sexo según año de medición Hogares biparentales con al menos 1 hijo/a 1990-2011 (CASEN)}

\begin{tabular}{|c|c|c|c|c|c|c|}
\hline \multirow{2}{*}{ CASADOS/AS } & \multicolumn{4}{|c|}{ Sexo } & \multirow{2}{*}{ Total } & \multirow{2}{*}{$\%$} \\
\cline { 2 - 5 } & Hombre & $\%$ & Mujer & $\%$ & & \\
\hline CASEN 1990 & 1.838 .397 & 98,0 & 37.896 & 2,0 & 1.876 .293 & 100,0 \\
\hline CASEN 2000 & 1.995 .116 & 96,7 & 67.163 & 3,3 & 2.062 .279 & 100,0 \\
\hline CASEN 2009 & 1.752 .541 & 90,7 & 179.095 & 9,3 & 1.931 .636 & 100,0 \\
\hline CASEN 2011 & 1.566 .129 & 86,8 & 237.849 & 13,2 & 1.803 .978 & 100,0 \\
\hline
\end{tabular}

Al observar el peso específico de los/as jefes de hogar casados de los hogares biparentales con al menos un hijo/a con el conjunto de los hogares entre 1990 y 2011, se constata una disminución del 7,7 de los jefes de hogar hombres y un incremento del 6,3\% en las mujeres (Cuadro $\mathrm{N}^{\circ}$ 9)

\section{Cuadro No9}

Proporción de jefes de hogares biparentales con al menso 1 hijo en relación al conjunto de jefes de hogares por sexo según año de medición 1990-2011 (CASEN)

\begin{tabular}{|r|r|r|r|}
\hline & \multicolumn{2}{|c|}{ Sexo } & \multirow{2}{*}{$\%$} \\
\cline { 1 - 2 } & Hombre & Mujer & \\
\hline CASEN 1990 & 86,1 & 69,9 & 85,7 \\
\hline CASEN 2000 & 85,8 & 79,3 & 85,6 \\
\hline CASEN 2009 & 80,0 & 74,0 & 79,4 \\
\hline CASEN 2011 & 78,4 & 76,2 & 78,1 \\
\hline
\end{tabular}


Polis, Revista Latinoamericana, Volumen 13, No 37, 2014

\section{Convivientes}

La proporción de jefes/as de hogar convivientes del conjunto de hogares se incrementó en el período. Ésta fue mayor entre los/as jefes/as de hogares biparentales con al menos un hijo.En el conjunto de los hogares, el año 1990, sólo el 6,3\% de los/as jefes de hogar era conviviente, valor que se incrementó al 15,0\% el 2011. Entre los/as jefes/as de hogares biparentales con al menos un hijo, esta proporción subió del 8,0\% al 23,6\%.

Al desagregar la información de jefes de hogar convivientes para el conjunto de hogares, según el sexo del/a jefe de hogar, se constata que el crecimiento de mujeres convivientes es mayor al de los varones entre 1990 y 1211. La proporción de mujeres se incrementa del 1,5\% al 9,5\%, casi 6 veces. Entre los varones ese crecimiento fue algo mayor al doble, del 7,6\% al $18,5 \%$ (Cuadro $\left.\mathrm{N}^{\circ} 10\right)$.

\section{Cuadro $\mathrm{N}^{\circ} 10$ \\ Jefes de hogar convivientes de todos los hogares por sexo según año de medición 1990-2011 CASEN}

\begin{tabular}{|c|c|c|c|c|c|c|}
\hline \multirow{2}{*}{$\begin{array}{c}\text { CONVIVIENTE } \\
\text { PAREJA }\end{array}$} & \multicolumn{4}{|c|}{ Sexo } & \multirow{2}{*}{ Total } & \multirow{2}{*}{$\%$} \\
\cline { 2 - 5 } & Hombre & $\%$ & Mujer & $\%$ & & \\
\hline CASEN 1990 & 192.095 & 7,6 & 9.449 & 1,5 & 201.544 & 6,3 \\
\hline CASEN 2000 & 370.112 & 12,4 & 56.651 & 6,3 & 426.763 & 10,9 \\
\hline CASEN 2009 & 514.515 & 16,4 & 152.021 & 9,8 & 666.536 & 14,2 \\
\hline CASEN 2011 & 561.796 & 18,5 & 183.414 & 9,5 & 745.210 & 15,0 \\
\hline
\end{tabular}

En los hogares biparentales con al menos 1 hijo/a los hombres jefes de hogar convivientes casi se triplican -del 7,8\% al 21,3\%- y entre las mujeres más que se duplican -del 15,8\% al 35,9\%) (Cuadro № 11).

\section{Cuadro $\mathrm{N}^{\circ} 11$ \\ Jefes de hogar convivientes de hogares biparentales con al menos 1 hijo/a por sexo según año de medición 1990-2011 CASEN}

\begin{tabular}{|c|c|c|c|c|c|c|}
\hline \multirow{2}{*}{$\begin{array}{c}\text { CONVIVIENTE O } \\
\text { PAREJA }\end{array}$} & \multicolumn{4}{|c|}{ Sexo } & \multirow{2}{*}{ Total } & \multirow{2}{*}{$\%$} \\
\cline { 2 - 6 } & Hombre & $\%$ & Mujer & $\%$ & & \\
\hline CASEN 1990 & 156.403 & 7,8 & 7.136 & 15,8 & 163.539 & 8,0 \\
\hline CASEN 2000 & 297.688 & 13,0 & 44.925 & 40,1 & 342.613 & 14,2 \\
\hline CASEN 2009 & 408.532 & 18,9 & 108.217 & 37,7 & 516.749 & 21,1 \\
\hline CASEN 2011 & 424.514 & 21,3 & 133.140 & 35,9 & 557.654 & 23,6 \\
\hline
\end{tabular}


El peso específico de jefes de hogar convivientes, en el conjunto de los jefes de hogar, es decreciente en el período, pasa de $81,1 \%$ al $74,8 \%$, entre 1990 y 2011. La misma tendencia se observa entre hombres y mujeres (Cuadro $\left.\mathrm{N}^{\circ} 12\right)$.

\section{Cuadro $\mathrm{N}^{\circ} 12$ \\ Jefas de hogar convivientes de hogares biparentales con al menos 1 hijo/a en relación al conjunto de hogares por sexo según año de medición 1990-2011 CASEN}

\begin{tabular}{|r|r|r|r|}
\hline & \multicolumn{2}{|c|}{ Sexo } & \multicolumn{1}{c|}{$\%$} \\
\hline & Hombre & \multicolumn{1}{c|}{ Mujer } & \\
\hline CASEN 1990 & 81,4 & 75,5 & 81,1 \\
\hline CASEN 2000 & 80,4 & 79,3 & 80,3 \\
\hline CASEN 2009 & 79,4 & 71,2 & 77,5 \\
\hline CASEN 2011 & 75,6 & 72,6 & 74,8 \\
\hline
\end{tabular}

\section{Número de hijos por hogar}

Se constata en el período 1990-2011 una disminución del número de hijos en el conjunto de los hogares y en los hogares biparentales con al menos un hijo/a (Cuadro $\mathrm{N}^{\circ} 15$ ).

En el conjunto de los hogares, se incrementó el porcentaje de hogares con un hijo/a en las últimas dos décadas, del 46,3\% al 59,4\% entre 1990 y 2011. Se redujo la proporción con dos, tres y más de tres hijos/as. Cada vez es menor la proporción del conjunto de hogares con más de un hijo; el año 1990 era de 53,7\%para descender el 40,5\% el 2011. El porcentaje en que se incrementó el conjunto de hogares con un hijo/ a, se redujo en los que tenían más de 2 (Cuadro $\mathrm{N}^{\circ} 13$ ).

La misma tendencia se observa en los hogares biparentales con al menos un hijo/a, aunque proporcionalmente tienen más hijos que el conjunto de los hogares. Crece la proporción de hogares con un y dos hijos entre 1990 y 2011 (13,4\%). Pero decrece en una proporción semejante los que tiene más de dos hijos (13,6\%) (Cuadro $\left.\mathrm{N}^{\circ} 13\right)$.

Los hogares biparentales con tres o más hijos disminuyeron su presencia de más de un tercio a algo menos de un cuarto (Cuadro $\mathrm{N}^{\circ}$ 13). 


\section{Cuadro $\mathrm{N}^{\circ} 13$}

Jefaturas de todos los hogares y hogares biparentales con al menos 1 hijo/a por \% de número de hijo/a/s según año de medición 1990-2011 CASEN

\begin{tabular}{|l|c|c|c|c|c|c|c|c|}
\hline & \% con 1 & \% con 2 & \% con 3 & $\begin{array}{c}\text { \% con + de } \\
\text { 3 }\end{array}$ & \% con 1 & \% con 2 & \% con 3 & $\begin{array}{c}\text { \% con + de } \\
\text { 3 }\end{array}$ \\
\hline CASEN 1990 & 28,2 & 35,1 & 22,5 & 14,3 & 46,3 & 26,6 & 16,5 & 10,6 \\
\hline CASEN 2000 & 30,6 & 37,6 & 21,9 & 10,0 & 49,4 & 27,8 & 15,7 & 7,1 \\
\hline CASEN 2009 & 36,0 & 38,8 & 18,8 & 6,4 & 57,3 & 26,1 & 12,3 & 4,2 \\
\hline CASEN 2011 & 37,9 & 38,8 & 17,3 & 5,9 & 59,4 & 25,9 & 10,9 & 3,7 \\
\hline
\end{tabular}

\section{Número de personas por hogar}

Entre 1990 y 2011, para el conjunto de los hogares se constata un incremento de los hogares con un máximo de tres miembros, y una disminución de los que tienen más de 3 . Los hogares con 1 sola persona se subieron del 6,9\% al 11,3\% en ese período (Cuadro $N^{\circ} 14$ ).

\section{Cuadro $\mathrm{N}^{\circ} 14$}

Número de personas por hogar del conjunto de los hogares por número de personas según año de medición 1990-2011 CASEN

\begin{tabular}{|l|c|c|c|c|c|c|c|c|}
\hline & \multicolumn{7}{|c|}{ Número de personas en el hogar } \\
\hline & \% con 1 & \% con 2 & \% con 3 & \% con 4 & \% con 5 & \% con 6 & \% con+ de 6 & Total \\
\hline CASEN 1990 & 6,9 & 13,8 & 19,8 & 23,6 & 17,5 & 9,0 & 9,4 & 100,0 \\
\hline CASEN 2000 & 8,0 & 15,1 & 20,5 & 24,8 & 16,8 & 8,0 & 6,9 & 100,1 \\
\hline CASEN 2009 & 10,1 & 19,4 & 22,1 & 23,2 & 14,0 & 6,3 & 4,8 & 100,0 \\
\hline CASEN 2011 & 11,3 & 21,3 & 22,8 & 22,2 & 12,4 & 5,6 & 4,3 & 100,0 \\
\hline
\end{tabular}

En los hogares biparentales se observa un incremento en los hogares que tienen hasta cuatro integrantes, o sea la pareja y uno o dos hijos/as; sobre ese porcentaje disminuye la proporción. Comparativamente estos hogares tienen más integrantes que el conjunto de los hogares (Cuadro $\mathrm{N}^{\circ}$ 15).

\section{Cuadro $\mathrm{N}^{\circ} 15$}

Número de personas por hogar de los hogares biparentales con 1 hijo por número de personas según año de medición 1990-2011 CASEN

\begin{tabular}{|l|l|c|c|c|c|c|c|c|}
\hline & \multicolumn{7}{|c|}{ Número de personas en el hogar } \\
\hline & \% con 1 & \% con 2 & \% con 3 & \% con 4 & \% con 5 & \% con 6 & \% con+ de 6 & Total \\
\hline CASEN 1990 & & & 21,4 & 30,9 & 23,5 & 11,9 & 12,2 & 100,0 \\
\hline CASEN 2000 & & & 23,2 & 33,7 & 23,5 & 10,6 & 9,0 & 100,0 \\
\hline CASEN 2009 & & & 26,5 & 35,0 & 21,8 & 9,5 & 7,2 & 100,0 \\
\hline CASEN 2011 & & & 27,7 & 35,5 & 20,7 & 9,2 & 6,9 & 100,0 \\
\hline
\end{tabular}




\section{Edad de los/as jefes de hogar}

En estas dos décadas, para el conjunto de los hogares y para los hogares biparentales con al menos un hijo/a, aumentó proporcionalmente la edad de los jefes de hogar. Se incrementaron los tramos que van de 45 y más años.

Disminuyó la proporción de jefes de hogar con edades en los tramos de menores de 45 años, para el conjunto de los hogares. Entre 1990 y 2011 decreció en los tramos de 15 a 24 años (del 2,6\% a 2,1\%), de 25 a 36 años (del $25,6 \%$ al 14,2\%) y de 35 a 44 años (del 28,3\% al 25,2). La mayor disminución se dio en el tramo entre 25 y 34 años. En cambio aumentaron los hogares con jefes de hogar mayores de 44 años (Cuadro $\mathrm{N}^{\circ} 16$ ).

\section{Cuadro $\mathrm{N}^{\circ} 16$ \\ Jefes/as del conjunto de hogares por tramo de edad según año de medición. 1990-2011 CASEN}

\begin{tabular}{|c|c|c|c|c|c|c|c|}
\hline & $\mathbf{1 5}$ a 24 & $\mathbf{2 5 - 3 4}$ & $\mathbf{3 5 - 4 4}$ & $\mathbf{4 5 - 5 4}$ & $\mathbf{5 5 - 6 4}$ & $\mathbf{6 5}$ y más & Total \\
\hline CASEN 1990 & 3,6 & 25,6 & 28,3 & 22,0 & 12,8 & 7,8 & 100,0 \\
\hline CASEN 2000 & 1,9 & 18,7 & 33,0 & 25,3 & 12,9 & 8,2 & 100,0 \\
\hline CASEN 2009 & 1,6 & 13,1 & 27,5 & 29,9 & 17,0 & 10,9 & 100,0 \\
\hline CASEN 2011 & 2,1 & 14,2 & 25,2 & 30,2 & 17,8 & 10,5 & 100,0 \\
\hline
\end{tabular}

La misma tendencia se constata en la edad de los/as jefes de hogar en los hogares biparentales con al menos un hijo/a, y también se constata que la mayor reducción está en el tramo 25 a 34 años. Crecen asimismo los/ as mayores de 45 años y, especialmente, los que tienen 65 o más años (Cuadro $\mathrm{N}^{\circ} 17$ ).

\section{Cuadro $\mathrm{N}^{\circ} 17$ \\ Jefes/as de hogares biparentales con al menos 1 hijo por tramo de edad según año de medición. 1990-2011 CASEN}

\begin{tabular}{|c|c|c|c|c|c|c|c|}
\hline & $\mathbf{1 5}$ a 24 & $\mathbf{2 5 - 3 4}$ & $\mathbf{3 5 - 4 4}$ & $\mathbf{4 5 - 5 4}$ & $\mathbf{5 5 - 6 4}$ & $\mathbf{6 5}$ y más & Total \\
\hline CASEN 1990 & 3,8 & 20,2 & 22,7 & 20,3 & 16,5 & 16,7 & 100,0 \\
\hline CASEN 2000 & 2,2 & 16,0 & 26,4 & 22,1 & 15,2 & 18,3 & 100,0 \\
\hline CASEN 2009 & 2,1 & 11,3 & 20,4 & 24,2 & 18,4 & 23,6 & 100,0 \\
\hline CASEN 2011 & 2,5 & 12,3 & 18,9 & 24,0 & 18,4 & 23,8 & 100,0 \\
\hline
\end{tabular}

Como constata en la información presentada, a comienzos de la transición democrática (1990) los jefes de los hogares biparentales con al menos 1 hijo,eran, casi en su totalidad, hombres casados. Expresión de la familia conyugal, al menos en estos atributos además del domicilio común. Si se hubiese mantenido el criterio de estado civil y sexo de los jefes de hogar (hombres y casados) las series históricas indicarían una disminución 
mayor de esta configuración a la presentada para estas dos décadas. La creciente presencia de convivientes y mujeres en la jefatura es una demostración más de las transformaciones de esta familia.

\section{Discusión}

La consolidación de familia conyugal estuvo en el centro de las políticas públicas durante gran parte del siglo XX. Fue parte de la agenda de importantes actores, como el empresariado vinculado al capitalismo industrial y minero, los partidos obreros y de sectores medio, las centrales obreras, y la Iglesia Católica. Ella fue el eje de las políticas de conciliación trabajo - familia, para la reproducción sustentable de la fuerza de trabajo y de las familias. El estado de bienestar no se explica ni se entiende, en sus componentes y procesos principales, si está ausente la supremacía que tiene esta configuración familiar impulsada desde el Estado, sostenida con recursos públicos y privados, y con una legislación del trabajo que buscó darle sustentabilidad. Ordenó el ámbito de la vida privada y de la organización del trabajo que estuvo vigente, al menos, hasta la década del 70.

Sobre la familia conyugal se puso las esperanzas de un avance significativo en el proceso civilizatorio -en términos de Elías- y fue calificada como aquella forma familiar que permitía sintetizar los atributos que habían sido pregonados por décadas desde la Revolución Francesa, pero que no lograron antes su concreción. Es en el siglo XX cuando se consolida esta configuración familiar, por las condiciones culturales, materiales, religiosas y subjetivas que se alcanzan, y por las agendas y actorías de los agentes sociales recién mencionados que permiten: derecho a decidir libremente sobre la pareja, lazo amoroso en el centro del contrato, vínculo/alianza para toda la vida, complemento de la pareja -una en lo crianza y otro en el trabajo productivo-, contrato de trabajo para que el hombre proveer, apoyos y subsidios del Estado para mantener a las mujeres en el hogar y en la crianza. La familia conyugal es uno de los pilares del orden de género del capitalismo industrial; consolida la división sexual del trabajo, profundiza la separación entre lo público y lo privado; en ella deposita la responsabilidad de la reproducción de la fuerza de trabajo, y estructura lo que corresponde a hombres y mujeres -los "roles sexuales".

Pero la sustentabilidad de esta configuración familiar comienza a debilitarse al menos desde fines de la década del 70’, cuando los requerimientos que la originaron, a fines del siglo XIX y comienzos del XX, dejaron de ser tales. Las políticas de policías de la familia devinieron en políticas focalizadas para subsidiar núcleos familiares indigentes y pobres, en particular aquellos encabezados por una mujer -los que presentan las mayores carencias-; las demandas del capitalismo industrial quedaron en la historia, con las transformaciones en la organización del trabajo la producción capitalista globalizada comenzó a demandar trabajadores para tareas específicas por tiempos determinados, con contratos crecientemente tercerizados y subcontratos; el magisterio y las enseñanzas de la Iglesia Católica perdie- 
ron fuerza, particularmente en la vida familiar, incluso los matrimonios religiosos disminuyeron en una proporción similar o mayor a los civiles, según fuentes del Episcopado; y finalmente, el matrimonio por amor para toda la vida, con roles sexuales asignados al hombre y a la mujer, se fue resquebrajando, disolviendo como lo muestran los datos recién analizados.

Es así, que lo que se ha descrito como la “crisis de la familia” no es sino la crisis de la familia conyugal, aquella configuración que se transformó en la segunda mitad del siglo XX en la "familia normal, tradicional”, con los atributos que han sido mencionados a lo largo de este artículo.

Durante las dos décadas de la transición democrática (1990-2011) esta configuración familiar ha ido perdiendo relevancia y peso, en relación al conjunto de los núcleos familiares, como se constata en los datos presentados; ya no está en el centro de las políticas públicas ni tampoco en las de los actores que tienen capacidad de decidir sobre ésta; salvo la persistente opinión moral y condena de la jerarquía de la Iglesia Católica y algunos grupos conservadores de los procesos en curso.

La información reprocesada de las bases de datos de la Encuesta Casende los años 1990, 2000, 2009 y 2011 permite sostener, a lo menos, lo siguiente

- que la familia conyugal dejó de ser la configuración mayoritaria en el país; su importancia relativa ha disminuido considerablemente y,de mantenerse, la tendencia histórica sería a una mayor pérdida.

- que otras configuraciones familiares, por el contrario, han tenido un crecimientos sostenido -madres/padres con hijos, abuelos con nietos, familias extendidas por la reincorporación de hijos/nietos a esos núcleos, núcleos de una sola persona.

- que disminuyó la proporción de varones como jefes de hogar tanto para el conjunto de los hogares, como para los biparentales, lo que pone en entredicho uno de los atributos principales de la familia conyugal: la división sexual del trabajo, el rol de proveedor y autoridad de la familia del hombre y de crianza para las mujeres.

- que decreció sistemáticamente la proporción de jefes de hogar casados, tanto para el conjunto de los hogares como para los biparentales con al menos 1 hijo/a, y se incrementó la de convivientes. El matrimonio civil pierde importancia y no es requerido por una proporción creciente de las parejas que dan origen a nuevas familias. - que las familias conyugales (hogares biparentales) tienen más hijos que las otras configuraciones, en un proceso en el que el conjunto de los núcleos familiares tiene cada menos hijos

- que están integradas por más miembros, que los otros núcleos familiares que viven en un mismo hogar.

- que la edad de los jefes de hogar, en las familias conyugales aumenta sistemáticamente, al igual que las del conjunto de configuraciones familiares, en la medida que la información de hogares biparentales se pueda vincular con la de esta configuración. 
En la búsqueda de hipótesis para explicar la desarticulación del orden familiar “normal” en estas últimas décadas y el desmoronamiento de la familia conyugal, es necesario tener presente, a lo menos, tres procesos que van a la par y contribuyen a ello. El primero, se ha seguido aplicado el principio de la subsidiaridad del Estado en la transición hacia la democracia (1990-2011). Cuando las familias requieren recursos que estén más allá de su capacidad -las más pobres-, el Estado los pone a su disposición en la medida de su capacidad y de la agenda de quienes gobiernan; el Estado es subsidiario en educación, salud, vivienda -por señalar algunos sectores- y entrega recursos a terceros privados que prestan esos servicios con financiamiento público, de lo que obtienen una “justa ganancia”, lucro.

El segundo, los cambios en la organización del trabajo y la precarización, particularmente entre los sectores tercerizados y de subcontratados. El tercero, y muy importante, los cambios en la condición de la mujer: su reconocimiento de "humana”, sus actorías y agendas políticas, con reconocimientos y derechos que el Estado está obligado a proteger, el empoderamiento de su cuerpo, el acceso a educación de calidad y al mercado del trabajo, proyectos de vida más allá de la maternidad, capacidad de decisión acompañada de recursos que le permiten autonomía económica, incluso una vez casada o con vida en pareja.

Si las políticas del Estado subsidiario, la organización del trabajo del capitalismo globalizado y la modernización tardía de la condición de la mujer al acceso pleno de sus derechos y actoría, han desarticulado el orden familiar y de género, o han profundizado los procesos que ya venía de antes, la pregunta que surge es ¿cuál es el orden familiar y de género que se busca construir? ¿Qué configuraciones de familia/s es/son la/s que se trata de fortalecer? Según los datos que hemos presentado, definitivamente, no ha sido la familia conyugal; por el contrario los recursos públicos y procesos señalados han inducido a una diversidad de configuraciones familiares que, a diferencia de las políticas de conciliación trabajo - familia de antaño, han precarizado las condiciones de la vida familiar y han acentuado las diferencias e inequidades entre familias, en razón de los recursos que poseen ¿Cómo articular a actores con intereses tan diversos para buscar un nuevo orden familiar y de género que concilie trabajo y familia e incentive configuraciones familiares sustentables que tengan en el centro el reconocimiento de sus derechos, la calidad de vida y felicidad de sus miembros?

Durante las dos décadas recientes no ha habido un debate sobre qué está pasando con "la familia” qué es lo que se busca, hacia adónde orientar la agenda y los recursos públicos, salvo la Comisión sobre la Familia creada durante el gobierno del Presidente Aylwin, que no llegó mucho más allá ¿Habrá llegado el momento de reiniciar ese debate? 


\section{Notas}

${ }^{1}$ Este artículo forma parte del Proyecto Fondecyt 1110553

${ }^{2}$ Los textos citados de Linton, Ralph y Parsons, Talcott son parteen la obra The family, que dirigió y preparó Ruth Nanda Anshen editada el año 1959 por Harper \& Brothers, publichers. Nueva York. La primera edición española, de parte de esta obra, la hizo Ediciones Península, Barcelona, España. La $8^{a}$ edición en español, de 1998, es la utilizada en este artículo; para las referencias de las citas se usa el año de la edición en inglés (1959) que las sitúa en el momento en que se desarrolla el debate.

3 Hallazgos que confirman estos aspectos se han obtenido en investigaciones desde los años 80 y 90 en Chile y en la región.

${ }^{4}$ Para la Encuesta CASEN el hogar es el "grupo de personas, parientes o no, que habitan la misma vivienda y tienen presupuesto de alimentación común o personas que viven solas. Puede ocurrir que uno o más hogares habiten una vivienda; sin embargo, un hogar no puede habitar más de una vivienda” (http:// www.ministeriodesarrollosocial.gob.cl/casen/definiciones/)

5 “Núcleo Familiar: Un hogar puede tener uno o más núcleos familiares. Un núcleo puede estar constituido por un matrimonio o unión de hecho sin hijos o hijastros o por un matrimonio o unión de hecho o un jefe (a) de familia con hijos o hijastros de cualquier estado civil, siempre y cuando estén solos, esto es, sin cónyuge o conviviente o hijos o hijastros. En ambos casos, el núcleo puede también estar constituido por menores de 18 años, parientes o no parientes, que dependan económicamente de la pareja o del jefe de familia y que no tengan ascendientes directos en el hogar y por otros parientes que no tengan ascendientes, descendientes o pareja viviendo en el hogar y que no puedan vivir solos, como por ejemplo personas de edad o con discapacidad” (http://www.ministeriodesarrollosocial.gob.cl/casen/definiciones/)

${ }^{6}$ Se tomó las dos últimas mediciones, 2009 y 2011, por el debate que se ha dado en torno a la toma de los datos y codificación de algunas preguntas de esta última encuesta. 


\section{Bibliografía}

Arriagada, Irma (2007), “Transformaciones familiares y políticas de bienestar en América Latina”. En Arriagada, Irma (cord) (2007), Familias y políticas públicas en América Latina: una historia de desencuentros. CEPAL, UNFPA, Santiago de Chile.

Beck, Ulrich y Elizabeth Beck-Gernsheim (2001), El normal caos del amor. Las nuevas formas de la relación amorosa. Paidos. Buenos Aires.

Ídem (2012), Amor a distancia. Las nuevas formas de vida en la era global.Paidos. Buenos Aires.

Becker, Gary (1987), Tratado sobre la familia. Alianza Universidad. Madrid.

Covarrubias, Paz; Mónica Muñoz y Carmen Reyes (1988), La pareja ¿encuentro o desencuentro?, Ediciones Universidad Católica de Chile. Santiago de Chile.

Connell, R. (2009), “Dentro de la torre de cristal: la construcción de las masculinidades en el mundo del capital financiero”. En Olavarría, José (ed.) (2009), Masculinidades y globalización. Trabajo y vida privada, familias y sexualidades. UAHC, CEDEM, Red de Masculinidad/es. Santiago de Chile.

Coontz, Stephanie (2006), Historia del matrimonio. Cómo el amor conquistó el matrimonio. Gedisa Editorial. Barcelona.

Díaz, Ximena, Lorena Godoy y Antonio Stecher (2005), Significados del trabajo, identidad y ciudadanía. La experiencia de hombres y mujeres en un mercado flexible. CEM Cuadernos de Investigación $N^{\circ} 3$. Santiago de Chile.

Donzelot, Jacques (1979), La policía de las familias. Editorial Pre-textos, Valencia.

Durán, María de los Ángeles (2008), “El futuro de las familias”. En Arriagada, Irma (ed) (2008), Futuro de las familias y desafíos para las políticas públicas, CEPAL, UNIFEM, UNFPA. Santiago de Chile.

Esteinou, Rosario (2008), La familia nuclear en México: lecturas de su modernidad. Siglos XVI al XX. CIESA - Porrúa. México D.F.

Fuller, Norma (2005), “Identidades en tránsito: Feminidad y masculinidad en el Perú actual”. En Valdés, Ximena y Teresa Valdés (eds) (2005), Familia y vida privada ¿Transformaciones, tensiones, resistencias y nuevos sentidos?, FLACSO/ CEDEM /UNFPA. Santiago de Chile.

García, Brígida y Orlandina de Olivera (2006), Las familias en el México 
Metropolitano: visiones femeninas y masculinas. El Colegio de México. México D.F.

Goody, Jack (2001), La familia europea. Editorial Crítica, Barcelona,.

Gubbins, Verónica, Brown, Francisca y Bagnara, Andrea (2003), “Familia: innovaciones y desafíos. Las familias chilenas en la década 1992-2002”. En Tironi, Eugenio et. al. (eds) (2003), Cuánto y cómo cambiamos los chilenos. Balance de una década. Censos 1992-2002. Publicaciones del Bicentenario. Santiago de Chile.

Hutchison, Elizabeth (1995), “La defensa de las ‘Hijas del Pueblo’. Género y política obrera en Santiago a principios de siglo”. En Godoy, Lorena et al. (eds) (1995), Disciplina y desacato. Construcción de la identidad en Chile. Siglos XIX y XX. SUR/CEDEM, Santiago de Chile.

Jelin, Elizabeth (2010), Pan y afectos: la transformación de las familias. Fondo de Cultura Económica. Buenos Aires.

Linton, Ralph (1959 edición original. $19701^{\text {a }}$ edición en español, 8a ed. 1998), "Introducción, la historia natural de la familia”. En Fromm, Erich, Max Horkheimer, Talcott Parsons y otros (1998), La familia. Ediciones Península. Barcelona.

Mauro, Amalia, Lorena Godoy y Virginia Guzmán (2001), Trabajo y relaciones de género: percepciones y prácticas de los varones. CEM. Santiago de Chile.

Méda, Dominique (2001), Les temps des femmes. Pour un nouveau partage des rôles. Flammarion, Paris.

Morandé, Pedro (1998) Familia y sociedad. Editorial Universitaria. Santiago de Chile.

Morant Deusa, Isabel y Mónica Bolufer Peruga (1998), Amor, matrimonio y familia. La construcción histórica de la familia moderna. Editorial Síntesis. Madrid.

Olavarría, José (2001), Y todos querían ser (buenos) padres. FLACSO. Santiago de Chile.

Ídem (2008), “Distribución del trabajo en las familias y (nuevas) masculinidades”.En Arriagada, Irma (ed) (2008), Futuro de las familias y desafíos para las políticas públicas, CEPAL, UNIFEM, UNFPA. Santiago de Chile.

Ídem (2009), "Hombres y trabajo en las identidades y en las relaciones de género”. En Zicavo, Nelson (2009), La familia en el siglo XXI. Investigaciones y reflexiones desde América Latina. Universidad del Bío-Bío. Concepción, Chile. 
Ídem (2009), “Globalización, género y masculinidades. Las corporaciones transnacionales y la producción de productores”. En Olavarría, José (ed) (2009), Masculinidades y globalización. Trabajo y vida privada, familias y sexualidades. UAHC, CEDEM, Red de Masculinidad/es. Santiago de Chile.

Oyarzún, Kemy (2005), “Ideologema de la familia: género, vida privada y trabajo en Chile 2000-2003”. En Valdés, Ximena y Teresa Valdés (eds.) (2005), Familia y vida privada ¿Transformaciones, tensiones, resistencias y nuevos sentidos?, FLACSO/ CEDEM /UNFPA. Santiago de Chile.

Rodó, Andrea y Ximena Valdés (eds.) (1995), “Aproximaciones a la familia”. En Proposiciones N²6. Ediciones Sur. Santiago de Chile.

Romero, Luis Alberto (1997), ¿Qué hacer con los pobres? Elite y sectores populares en Santiago de Chile. 1840-1895. Editorial Sudamericana. Buenos Aires.

Rosenblatt, Karin (1995), "Masculinidad y trabajo: el salario familiar y el estado de compromiso, 1930-1950”. En Proposiciones N²6, Aproximaciones a la familia, SUR Ediciones. Santiago de Chile.

Ídem (1995), "Por un hogar bien constituido. El Estado y su política en los Frentes Populares”. En Godoy, Lorena et. al. (eds.) (1995), Disciplina y desacato. Construcción de identidad en Chile, siglos XIX y XX. Colección Investigadores Jóvenes, Ediciones SUR-CEDEM. Santiago, Chile.

Stone, Lawrence (1989), Familia, sexo y matrimonio en Inglaterra 15001800. Fondo de Cultura Económica. México D.F.

Valdés, Ximena (2008), "Notas sobre la metamorfosis de la familia en Chile”. En Arriagada, Irma (ed)(2008), Futuro de las familias y desafíos para las políticas públicas, CEPAL, UNIFEM, UNFPA. Santiago de Chile.

Valdés, Teresa (2008), “Aprendizajes, aspectos pendientes y propuestas para las políticas hacia las familias”. En Arriagada, Irma (ed)(2008), Futuro de las familias y desafíos para las políticas públicas, CEPAL, UNIFEM, UNFPA. Santiago de Chile.

Valdés, Teresa y Ximena Valdés (eds.) (2005), Familia y vida privada ¿Transformaciones, tensiones, resistencias o nuevos desafíos? CEDEM/ FLACSO. Santiago de Chile.

Valenzuela, Samuel, Eugenio Tironi y Timothy Scully (eds) (2006), EL eslabón perdido. Familia, modernización y bienestar en Chile. Taurus. Santiago de Chile.

Viveros, Mara (2002), De quebradores y cumplidores. Sobre hombres, masculinidades y relaciones de género en Colombia. CES. Universidad Nacional de Colombia. Bogotá, Colombia. 
José Olavarría A.

Wainerman, Catalina (2005), La vida cotidiana en las nuevas familias ¿una revolución estancada?, Lumiere. Buenos Aires, Argentina.

Recibido: 19.03.2013

Aceptado: 20.08.2013 